\title{
When the Wheels Touch Earth and the Flight is Through, Pilots Find One Eye is Better Than Two
}

\author{
Brian Valimont ${ }^{\alpha}$, John A. Wise*, Troy Nichols, Carl Best, John Suddreth, \& Frank Cupero \\ Honeywell International, 21111 N. 19 ${ }^{\text {th }}$ Ave. Phoenix, AZ 85027-2700 USA
}

\begin{abstract}
This study investigated the impact of near to eye displays on both operational and visual performance by employing a human-in-the-loop simulation of straight-in ILS approaches while using a near to eye (NTE) display. The approaches were flown in simulated visual and instrument conditions while using either a biocular NTE or a monocular NTE display on either the dominant or non dominant eye. The pilot's flight performance, visual acuity, and ability to detect unsafe conditions on the runway were tested.
\end{abstract}

Keywords: Near-to-eye display, far vision acuity, binocular

\section{INTRODUCTION}

The use of collimated virtual displays (e.g., HUDs) by pilots has become more commonplace in aircraft. After decades of development and deployment by all branches of the military, FAA certified HUDs have become standard equipment on many airliners. The success of HUDs is leading researchers to consider other forms of collimated virtual displays; one form that shows promise is the near to eye display (NTE). An NTE is simply a virtual collimated display with the projection lenses placed close to and directly in front of one or both of the pilot's eyes. This type of display is generally mounted on some type of helmet. With the development of such a display has come several research questions.

Most obvious is the question of whether to place the projection lens in front of one or both eyes (monocular versus biocular) and are there any associated pilot performance effects that would influence that choice? Binocular rivalry is one potential concern during the use of a monocular display. Dominance/suppression phasing of binocular rivalry might be induced by a monocular head mounted display (HMD) and is certainly a consideration when answering the monocular versus biocular questions for NTE displays. In fact, a number of authors have already identified binocular rivalry as a potentially serious perceptual problem related to HMDs (e.g., ${ }^{1}$ Blackwood et al. and ${ }^{2}$ Peli). There also seems to be anecdotal evidence that pilots experience perceptual problems possibly related to binocular rivalry while using a monocular display. ${ }^{3}$ Rush et al. reported that some pilots stated that they resorted to closing one eye because of the difficulty switching attention between eyes while using a monocular display during flight operations.

In addition to the issue of binocular rivalry, general criticisms of collimated virtual displays that arose during HUD development must also be addressed to ensure that NTE displays are safe and effective for pilot use. One of the most noted criticisms is that pilots have a tendency to focus on the HUD combining glass instead of the outside world. It was theorized that the human eye does not focus well at optical infinity when viewing collimated virtual images, but have a tendency to focus inward to resting accommodation at a distance of approximately arm's length $\left({ }^{4} \mathrm{Hull}, \mathrm{Gill}, \&\right.$ Roscoe; ${ }^{5}$ Roscoe, and ${ }^{6}$ Roscoe $)$. Their conclusion that the misaccommodation of the eye at optical infinity, and the associated loss of visual acuity, portends a performance degradation in the detection and identification of potential targets in the outside world.

However, a study reported by Wise \& Sherwin ${ }^{7}$ found no such visual effects when items in the near vision (e.g., HUD structure), were not in the visual field. Given that near to eye technology has no such structure in the near vision, the authors felt that it would seem reasonable to hypothesize that near-to-eye technology could help pull the users' eyes toward focusing at optical infinity rather than at or near resting accommodation.

\footnotetext{
${ }^{\ni}$ The authors would like to recognize both the financial and technical support provided by NASA Langley under contract NNL06AA05B

${ }^{\alpha}$ Brain Valimont is currently with SA Technologies

*John.A.Wise@Honeywell.com, phone: +1 (602) 436-5536, fax +1(602) 822-7000
} 
The goal of this study was to directly measure a pilot's ability to accommodate at optical infinity using an unobtrusive measure of visual acuity.

\subsection{Experimental Design}

The design of the NTE investigation was a completely within-subjects ( 3 x 4 x 4 x 12) experiment.

\subsection{Independent Variables}

The first variable included three levels of different NTE display configurations, biocular, monocular over dominant eye, and monocular over non-dominant eye. Each NTE display configuration was worn by every pilot through all types of meteorological conditions, and runway incursion variable levels. The second independent variable included in the study is the meteorological condition in which the approaches were flown. Four meteorological conditions were chosen: 1) day visual meteorological conditions (VMC), 2) night VMC, 3) day instrument meteorological conditions (IMC), and 4) night IMC. The last independent variable were runway incursion trials. This variable consisted of two levels. The first level was a clear active runway on which the pilots were able to land. The second level of the variable was the appearance of ground traffic on the approach end of the runway for which the pilot was cleared to land.

\subsection{Dependent Measures:}

Visual Acuity. Throughout each approach the pilot flew, Landolt rings were displayed at random time intervals. The Landolt rings, a classic measure of visual acuity, were used to measure the pilot's physical ability to accurately see and identify far vision targets. Landolt rings consist of a shape that resembles the letter $\mathrm{O}$ except that a notch is cut out of the circle (Figure1). The visual acuity test is a measure of whether the participant can identify the location of the notch according to a standard clock face (e.g., the notch is at 3 o' clock). Landolt rings were projected onto the simulation screen that displayed the outside world flight simulation. All Landolt rings were scaled for 20/20 visual acuity. Misses and correct identifications were recorded for every approach.

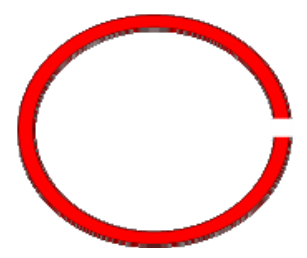

Figure 1. Example of a Landolt ring.

\section{METHOD}

\subsection{Participants}

Twelve certificated pilots participated in this investigation. Eleven of the 12 were Airline Transport Pilot (ATP) rated. The mean flight experience in terms of total flight time was 12,017.0 hours and the mean flight experience with heads-up displays (HUDs) was 2,275.0 hours. Six of the 12 pilots wore corrective lenses.

\subsection{Apparatus}

The testing apparatus consisted of the head mounted NTE display which was constructed by integrating two Microvision ${ }^{\circledR}$ Nomad $^{\mathrm{TM}}$ displays and a custom-built head mount which had an adjustment to accommodate differences in head size. The Nomad displays were mounted in such a way that an adjustment could be made to the displays to accommodate differences in inter-pupil distance. The displays had a hardwired control pad and internal menu structure which allowed the experimenter to make adjustment to the position of the symbology on the display to account for individual differences in dipvergence (see Figure 2).

Additional equipment used in the experiment included the Ascension ${ }^{\circledR}$ Phasor Bird ${ }^{\mathrm{TM}}$, which provided head tracking data to ensure the display imagery was stable as the pilot moved his head during the simulated approaches (see Figure 2). The simulated ILS approach into Chicago-Midway runway 4R was driven by Microsoft ${ }^{\circledR}$ Flight Simulator $\mathrm{X}^{\mathrm{TM}}$ and aircraft control was provided by an off-the-shelf control yoke. The heads down primary flight display (PFD) was displayed on a Honeywell ${ }^{\circledR} 1310$ LCD display. The screen onto which the flight simulation was projected was located at a distance from the pilot effectively equal to optical infinity (approximately 26 feet). 

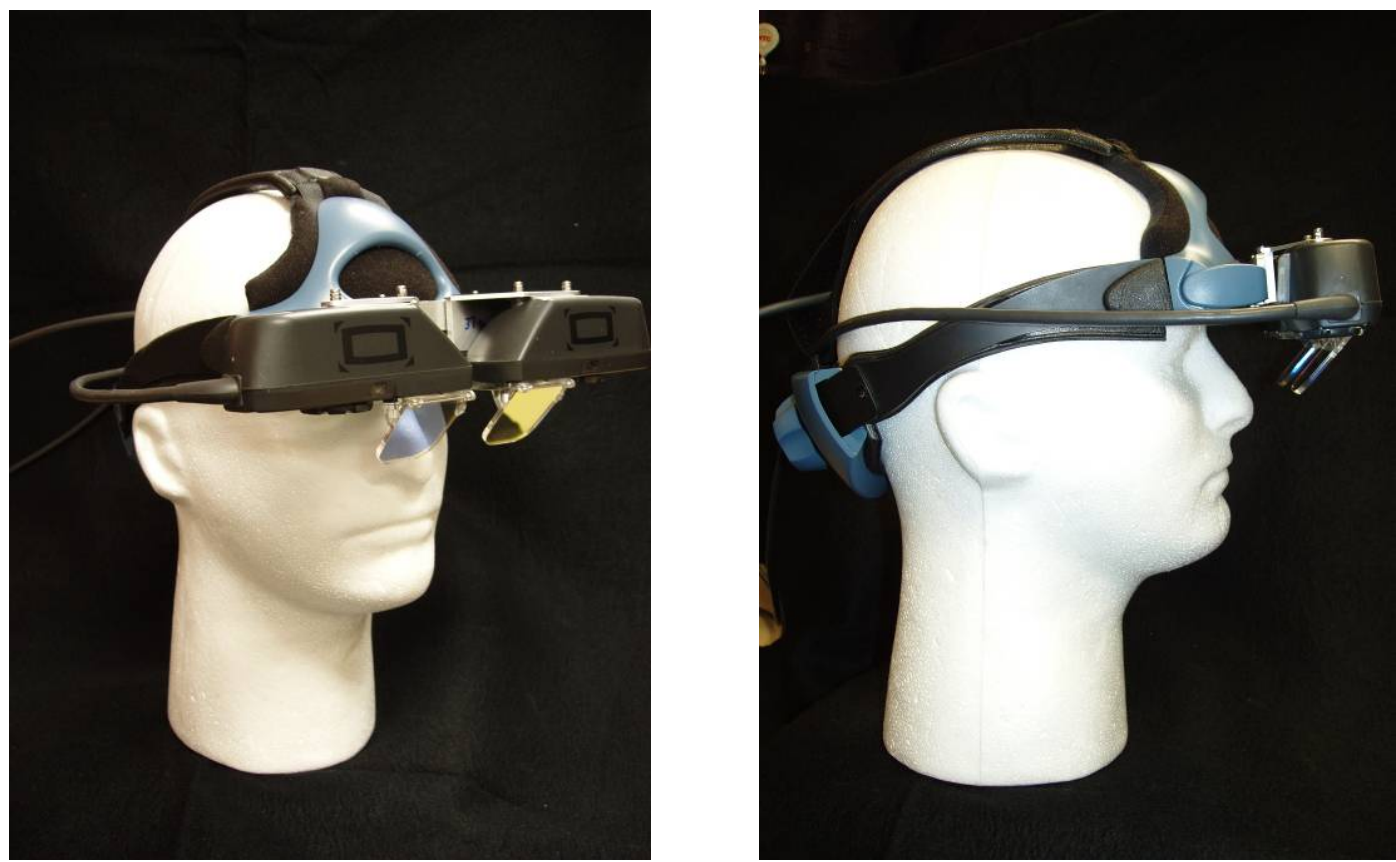

Figure 2. Photos of the experimental near to eye display worn by the participants.

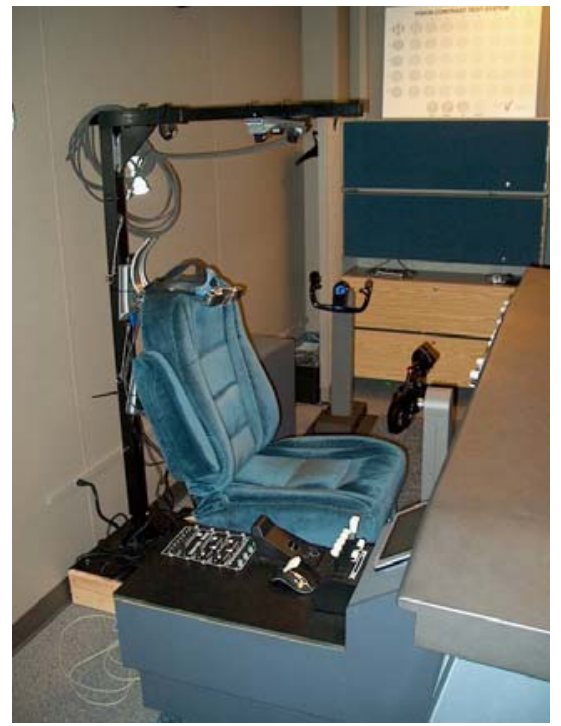

Figure 2. Photo of the head tracker and cockpit set-up.

\section{PROCEDURE}

Before the calibration of the NTE display was begun, pilots reviewed the informed consent form, asked if they had any questions and, if they agreed to participate, to sign the form. They were given a copy of the signed form.

Once the paper work was complete they were given a short instruction period where the basic purpose of the experiment, and the flight performance expected from them, was explained. They were also assigned their 
participant number, and told what responses were appropriate for the appearance of the Landolt rings. The last part of this initial element of the experiment was a test to determine the pilot's dominant eye. The eye dominant was tested through the classic "hole in the card test.” Participants were given an index card with a hole cut out in the center. They were told to focus on an object in the room through the hole in the card, while the card was held by the participant at arm's length. Then, while keeping the object in the center of the hole, they were instructed to bring the card back to their face. They then were told to close one eye at a time and asked which eye could still see the object through the hole in the card. The participant's dominant eye was recorded on a data collection sheet. The introductory portion of the experiment lasted approximately 30 minutes.

\subsection{NTE Display Calibration}

Pilots fitted themselves with the head mounted display and made adjustments until the HMD felt snug but comfortable. The experimenter then adjusted the NTE displays to match the participant's inter-pupil distance. Lastly, a calibration screen was presented on the NTE display and a calibration target was displayed on the simulator screen so that the experimenter and participant could make adjustments to the head tracker and accommodate differences in dipvergence, bringing the symbology shown on the NTE display into a converged focused picture. When pilots stated the symbology was properly focused and they no longer saw double imaging or ghosting, then calibration was complete. This portion of the experiment typically lasted between $20-30$ minutes.

\subsection{Experimental Session}

Each pilot flew four types of approaches (one approach contained one level of meteorological condition and one level of runway incursion event) through the use of a fixed-base flight simulator with each of the three display configurations for a total of 12 approaches in an experimental session. The order of the display configurations, different meteorological conditions, and the runway incursion variable was counterbalanced using a full Latin Square design, to insure that ordering effects did not confound the data and experimental results.

The following scenario is an example of a pilot's experience during one of the 12 simulated approaches. The pilot was placed in the flight simulator and, following calibration of the NTE display, the experimental session began with the five mile straight-in instrument landing system (ILS) approach to runway $4 \mathrm{R}$ at Chicago Midway airport. The flight began with the aircraft centered on the localizer for an ILS approach. Throughout the approach, Landolt rings were displayed at random time intervals at a fixed location in the outside field of view. Pilots were required to identify the appearance of the Landolt ring and the location of the Landolt ring notch (e.g., 4 o' clock). When the pilot captured the glideslope, he or she continued to identify the appearance of Landolt rings until breaking out of the cloud layer ${ }^{1}$. At this point, the flight simulation showed ground traffic taxiing onto the runway. Thepilot was expected to identify the hazardous situation and react appropriately.

\section{DATA ANALYSIS}

The nominal data of the Landolt ring correct identifications and misses were analyzed using a Fisher's Exact Test. Since some data cells of the experimental design contained values of less than five, it was determined that the Chi-Squared test was not the appropriate tool, rather a Fisher's Exact Test was the correct analysis in this case (Ott \& Longnecker, 2001). Where significant differences were found, further pairwise contrasts were performed using additional Fisher's Exact Test procedures.

\section{RESULTS}

Visual Acuity. A Fisher's Exact Test was used to compare the visual acuity of pilots. Results have shown a significant difference between the three display configurations $(p<0.001)$. Further pairwise comparisons using Fisher's Exact Test showed pilots correctly identified more Landolt Rings while wearing the monocular display over the dominant eye than while wearing the biocular display $(p<0.001)$. Pairwise comparisons also showed that pilots correctly identified more Landolt Rings while wearing the monocular display over the non-dominant eye than while wearing the biocular display $(p<0.001)$. No differences were found between the dominant eye and non-dominant eye monocular displays $(p=0.15)$ (see Figure 3$)$.

${ }^{1}$ This example illustrates a straight-in approach in IMC conditions. 


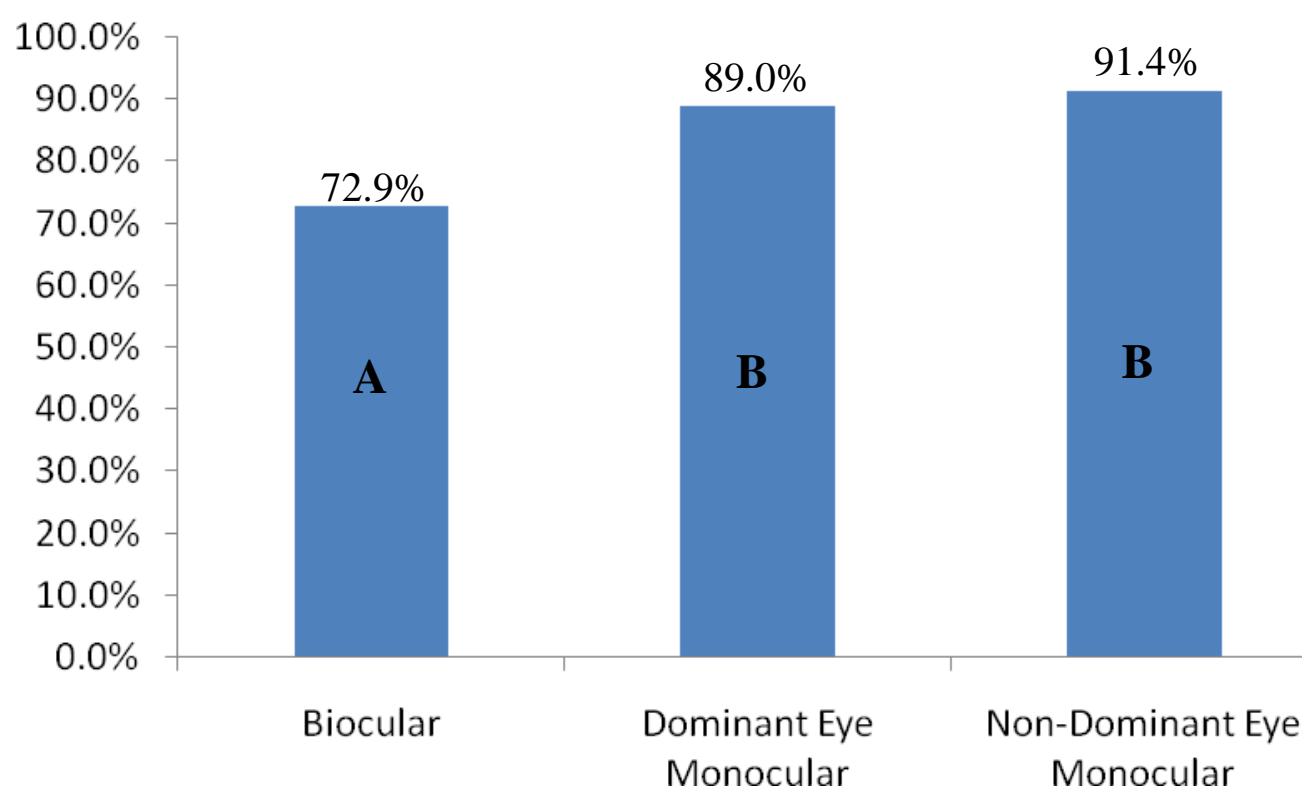

Figure 3. Mean identification accuracy of Landolt ring visual acuity test by display configuration. Different letters represent significant differences at the $p<0.05$ level.

The visual acuity data directly measured the pilot's ability to accurately detect, focus on, and identify a target at optical infinity. The results of this study directly contradict those of previous studies. ${ }^{4,5}$, 6 With accuracy scores of $72.9 \%$, 89.0\%, and $91.4 \%$ for the biocular, dominant eye monocular, and non-dominant eye monocular displays, respectively, the results of this study clearly indicate that not only do pilots have the physiological capability to accommodate between the near field and optical infinity, but they are able to utilize this capability very effectively when identifying potential targets at optical infinity. The visual acuity analysis of this study also revealed significant differences between the display configurations. Pilots had significantly greater visual acuity while wearing either of the monocular display configurations than when wearing the biocular display, with no differences found between the two monocular displays. This may be due to the unobstructed view of the outside world afforded to one eye by the monocular display, which may enable the pilot to more easily perceive and focus on outside visual targets.

This study also revealed one very important consideration for developing NTE displays: the pilot's visual acuity at optical infinity was significantly more accurate with a monocular display than a biocular display. The ability to accurately perceive and identify a potential target or conflict is not only a major safety advantage but it may also be an advantage in terms of solving the conflict in a way that is advantageous to the pilot's mission. Therefore, the evidence of this study supports an advantage of NTE monocular displays over their biocular counterpart.

\section{CONCLUSIONS}

This study investigated potential differences between monocular and biocular NTE displays. Binocular rivalry was a potential problem reported previously in the literature but did not seem to affect pilot performance within any data measures collected during this experiment. Furthermore, no subjective statements in the postexperiment interviews revealed either awareness of dominance/suppression phases of the eyes, trouble focusing on the flight symbology or the outside world visuals while wearing either of the monocular display configurations. This is not to say binocular rivalry was not present. It may not have reached a level that pilots were consciously aware of it nor affected their performance on any of the dependent measures. 


\section{REFERENCES}

[1] Blackwood, W.O., Anderson, T.R., Bennett, C.T., Corson, J.R., Endsley, M.R., Hancock, P.A., Hochberg, J., Hoffman, J.E., \& Kruk, R.V. Tactical displays for soldiers: Human factors considerations. Panel on Human Factors in the Design of Tactical Display Systems for the Individual Soldier, National Research Council, National Academy Press. (1997).

[2] Peli, E. Visual issues in the use of a head-mounted monocular display. Optical Engineering, 29(8), 883892. (1990).

[3] Rush, C.E., Verona, R.W., \& Crowley, J.S. Human factors and safety considerations of night vision systems flight using thermal imaging systems. Tech. Rep. 90-10, U.S. Army Aeromedical Research Laboratory. (1990).

[4] Hull, J.C., Gill, R.T., \& Roscoe, S.N. Locus of the stimulus to visual accommodation: Where in the world, or where in the eye. Human Factors, 24, 311-319. (1982).

[5] Roscoe, S.N. Bigness is in the eye of the beholder. Human Factors, 27, 615-636. (1985).

[6] Roscoe, S.N. The trouble with HUDs and HMDs. Human Factors Society Bulletin, 30(7), 1-3. (1987).

[7] Wise, J.A. \& Sherwin, G.W. An empirical investigation of the effect of virtual collimated displays on visual performance. Proceedings of the Fifth International Symposium on Aviation Psychology. Columbus, OH, 17-20 April. (1989).

[8] Ott, R.L., \& Longnecker, M. An Introduction to Statistical Methods and Data Analysis. Pacific Grove, CA: Duxbury. (2001). 